\title{
Evaluación prospectiva de un protocolo de actuación para la metatarsalgia por insuficiencia de primer radio: resultados de tratamiento conservador y quirúrgico
}

Prospective evaluation of an action protocol for metatarsalgia due to failure of the first radius: results of conservative and surgical treatment

\begin{abstract}
Borja Cerdá', Luke D. Cicchinelli², Laura Prats ${ }^{3,4}$, Josep Conde ${ }^{5}$ y Joan Viñas ${ }^{3,4}$
'Práctica privada, Departamento de Cirugía, Universitat de Lleida, España. ${ }^{2}$ Práctica privada, Vigo, España. ${ }^{3}$ Universitat de Lleida y Hospital Universitario Arnau de Vilanova, Lleida, España. ${ }^{4}$ nstitut de Recerca Biomèdica de Lleida. ${ }^{5}$ Departamento de Matemática, Universitat de Lleida, España
\end{abstract}

\section{Palabras clave:}

Corticoides metatarsalgia, factores de riesgo, insuficiencia del primer radio, osteotomía de Weil, protocolo, soportes plantares, tratamiento conservador, tratamiento quirúrgico, infiltraciones perilesionales.

\section{Resumen}

Introducción: La metatarsalgia por insuficiencia de primer radio es una de las patologías más frecuentes del pie, cuyo protocolo de tratamiento no está bien establecido. El objetivo de este estudio prospectivo es valorar un protocolo de actuación, y considerar diferentes factores etiológicos que influyen en la patología y los resultados del tratamiento.

Pacientes y método: Se valoraron pacientes diagnosticados de metatarsalgia por insuficiencia del primer radio, diagnosticados por radiología simple y estudio biomecánico, evaluando las presiones plantares y el dolor, función y alineación mediante la escala American Orthopedic Foot and Ankle Surgery (AOFAS) al inicio y después de cada tratamiento; aplicando el tratamiento con soportes plantares personalizados con barra metatarsal y extensión de Morton, a los 3 meses según la efectividad, infiltración con corticoides, evaluando al paciente a los 6 meses y, en caso de fracaso de los dos anteriores, se indicó el tratamiento quirúrgico utilizando la osteotomía de Weil. Seguimiento de cada a paciente a un año, observando qué variables pueden influir en la patología.

Resultados: La muestra final fue de 56 pacientes: 46 permanecieron asintomáticos mediante soportes plantares, 10 precisaron soportes plantares e infiltración con corticoides, de los cuales 4 dejaron de tener dolor y 6 , al ser inefectivos los anteriores tratamientos, fueron sometidos a la cirugía.

Discusión: Tres variables fueron significativas asociadas a la metatarsalgia antes de aplicar cualquier tratamiento (índice de masa corporal, patologías asociadas, inestabilidad de la articulación metatarsofalángica afectada) y la variable prevalente después del tratamiento de infiltración y cirugía fue cirugía previa.

Keywords:

Corticosteroids, metatarsalgia, risk factors, first ray insufficency, Weil osteotomy, protocol, insoles conservative treatment, surgical treatment, intralesional infiltrations.

Recibido: 01-05-2019 Aceptado: 13-10-2019

\section{Abstract}

Introduction: Metatarsalgia for first ray insufficiency is one of the most common pathologies of the foot, whose treatment protocol is not well established. The objective of this prospective study is to assess a protocol of treatment, and to consider different factors that influence the pathology and treatment results.

Patients and method: Patients diagnosed with metatarsalgia for first ray insufficiency diagnosed through simple radiology and biomechanical study were prospectively assessed, evaluating plantar pressures and pain, function and alignment using the American Orthopedic Foot and Ankle Surgery (AOFAS) scale at the beginning and after each treatment; first applying the treatment with personalized plantar supports with metatarsal bar, at 3 months according to effectiveness, infiltration with corticosteroids, evaluating the patient at 6 months and, in case of failure of the previous two, the treatment was indicated using Weil's osteotomy. Follow-up of each patient to one year, observing that variables can influence the pathology.

Results: The final sample was of 56 patients, of whom 46 remained asymptomatic by plantar supports, 10 patients needed plantar supports and infiltration with corticosteroids, of which 4 ceased to have pain and 6 patients the previous treatments were ineffective, they underwent surgery.

Discussion: Three variables were significant associated with metatarsalgia before applying any treatment (Body Mass Index, associated pathologies, affected metatarsal-phalagic joint instability) and the 2 prevalent variables after applying the infiltration and surgery treatment were previous surgery and insufficiency of first iatrogenic ray. 


\section{INTRODUCCIÓN}

La metatarsalgia de radios medios consiste en dolor en la zona de las cabezas de los metatarsianos centrales. Se caracteriza por la presencia de dolor en el antepié, por sobrecarga mecánica, produciendo una lesión por sobreuso y quedando afectados el segundo, tercero y cuarto metatarsianos ${ }^{1}$. La metatarsalgia puede tener múltiples etiologías producidas por un exceso de sobrecarga mecánica en la zona central del metatarso en el antepié. La distribución de las fuerzas puede variar con la actividad física, la edad, el calzado, la retracción de la musculatura posterior y la morfología del antepié2. Los factores biomecánicos explican el $90 \%$ de las causas de la metatarsalgia y las causas de la metatarsalgia están clasificadas en 3 grupos: primaria, secundaria e iatrogénica después de una cirugía de antepié ${ }^{3}$.

El desarrollo de la cirugía de antepié podría también contribuir a una mayor incidencia de metatarsalgia por causas iatrogénicas, sobre todo la cirugía del hallux valgus, ya que puede provocar un excesivo acortamiento o elevación del primer metatarsiano ${ }^{4,5}$.

Hoy en día, la cirugía como tratamiento para la metatarsalgia es controvertida. Hay muchos procedimientos que se pueden realizar para la intervención quirúrgica localizada, que siempre vendrá dirigida por los síntomas, los hallazgos físicos del paciente y la evaluación radiológica, ${ }^{6,7}$.

El presente estudio se centra únicamente en la metatarsalgia primaria, provocada por la insuficiencia de primer radio, que describió por primera vez Viladot como el síndrome de la insuficiencia de primer radio ${ }^{8,9}$. Esta se caracteriza por una disminución de la cantidad de carga que soporta la cabeza del primer metatarsiano, lo que puede provocar una sobrecarga del resto de las estructuras del antepié, generalmente el segundo y el tercer metatarsianos, en la estática y en la dinámica ${ }^{10,11}$. La etiología puede ser congénita, por un primer metatarsiano corto, por debilidad de las partes blandas en la articulación metatarsocuneana que no fijan el radio durante la marcha, por la supinación del antepié en el pie plano valgo, o puede responder a una etiología iatrogénica por acortamiento excesivo del primer radio en el tratamiento quirúrgico previo del hallux valgus ${ }^{12}$.

El tratamiento de la metatarsalgia puede ser médico o conservador $^{11,13-28}$, o quirúrgico ${ }^{12-14,29-31}$. El tratamiento conservador más indicado son los soportes plantares semirrígidos personalizados con una descarga en forma de barra retro capital, para descargar la zona metatarsal afectada y una pieza subcapital de Morton para provocar la carga del primer metatarsiano $^{32}$. Otros dos parámetros se consideran de importancia, ya que pueden contribuir también a la aparición de la metatarsalgia: la adaptación a medida del arco plantar del paciente y la neutralización de la articulación subastragalina, para evitar aplanamientos, valguismos y compensar las debilidades de partes blandas que pueden afectar al antepié ${ }^{33,34}$. La recuperación funcional, la fisioterapia, los masajes, los ejercicios pasivos, los ejercicios activos o los fármacos antinflamatorios sirven como tratamientos coadyuvantes. Sin embargo, estos tratamientos solo son útiles en los casos de reagudizaciones dolorosas, o como obligada rehabilitación postquirúrgica ${ }^{13}$. Las infiltraciones locales con corticoesteroides son un buen tratamiento, siempre que estén asociadas al uso de soportes plantares que descarguen la zona metatarsal previamente, en los casos donde, a pesar de la descarga, pueda persistir el edema articular, la inflamación tisular o de otros tejidos adyacentes ${ }^{11,18,19,35}$

El tratamiento quirúrgico está generalmente indicado para metatarsalgias recalcitrantes refractarias a los tratamientos conservadores ${ }^{30,37}$, consecuencia de metatarsianos largos con o sin deformidades del plano transversal digital, dedos cruzados y subluxaciones o luxaciones de la articulación metatarsofalángica. Estas son las indicaciones de este procedimiento, aunque también se ha utilizado para corregir deformidades reumáticas ${ }^{30,37}$. Existen diferentes técnicas para acortar y corregir la posición en plantar flexión del metatarsiano afectado, pero actualmente la más utilizada por su versatilidad y estabilidad es la osteotomía de Weil, con sus diversas modificaciones, descrita por el podiatra americano Lowell Weil. La osteotomía de Weil puede ser utilizada en uno o más metatarsianos de acuerdo con la complexidad de las deformidades del antepié y el patrón de longitud total de los metatarsianos adyacentes ${ }^{29,37-39}$

Aunque hay muchos estudios relacionados con la metatarsalgia, no queda claramente definido el valor de las diferentes variables asociadas a la patología y los factores pronósticos para determinar el fracaso o éxito de los tratamientos y el orden de actuación. El presente estudio pretende valorar un protocolo de tratamiento que tiene la finalidad de estudiar la efectividad de una actuación frente a la patología metatarsal por insuficiencia de primer radio, teniendo en cuenta diferentes factores de riesgo que puedan influir en la metatarsalgia por insuficiencia de primer radio, antes y después de cada tratamiento.

\section{PACIENTES Y MÉTODOS}

\section{Población de estudio}

Se realizó un estudio prospectivo de 24 meses de duración con pacientes diagnosticados de patología metatarsal central por insuficiencia de primer radio, recogidos en el Institut Mèdic del Peu y la Clínica de Ponent de Lleida (Cataluña, España). La toma de datos se llevó a cabo entre septiembre de 2016 y septiembre de 2017. Se realizó un seguimiento posterior de todos los pacientes durante un año, finalizando la toma de datos en septiembre de 2018.

Los criterios de inclusión fueron pacientes mayores de edad que padecían la sintomatología de metatarsalgia central por insuficiencia de primer radio. El diagnóstico de la metatarsalgia central por insuficiencia de primer radio se determinó por: la insuficiencia funcional y déficit de apoyo en el estudio baro- 
podométrico; la exploración en sedestación para examinar alteraciones musculoligamentosas, como la debilidad de partes blandas o hipermovilidad de las articulaciones del primer radio (metatarsocuneal y metatarsofalángica); y radiografía simple anteroposterior en carga, con la finalidad de confirmar e identificar el acortamiento del primer radio respecto al segundo y el resto de los metatarsianos que provocase poca carga en el primer metatarsiano durante situaciones de carga.

Se excluyeron aquellos pacientes con metatarsalgias provocadas por otras causas: longitud del segundo o tercer metatarsianos, deformidades congénitas de las cabezas metatarsales, acortamiento de los músculos gastrocnemios o del tríceps sural, un pie equino, un pie cavo, así como anormalidades del retropié que pueden afectar la posición del antepié. También fueron excluidos aquellos pacientes menores de edad, alérgicos a los corticoides o pacientes con artritis reumatoide, gota o psoriasis, alteraciones neurológicas como Charcot-Marie-Tooth, enfermedad de Freiberg, diabéticos con mal control metabólico o todos aquellos que voluntariamente decidieron rechazar entrar en el estudio. El estudio pasó por el comité de ética de la Facultad de Medicina de la Univesitat de Lleida, con número de CEIm CEIC-2156, del Hospital Universitari Arnau de Vilanova de Lleida.

\section{Medición de variables}

Se realizaron las siguientes mediciones:

a) Estudio biomecánico de la marcha mediante plataforma de presiones baropodométricas para observar las presiones plantares con la finalidad de identificar la coincidencia de la zona dolorosa en los metatarsianos centrales con la de más presión en estática y en dinámica, y la insuficiencia funcional de apoyo del primer metatarsiano.

b) Exploración física del paciente en sedestación, estática y dinámica: se examinó el tipo de pie mediante el Foot Posture Index (FPI) para conseguir una fácil cuantificación de la postura del pie en estática. Se realizó también el test de Lunge para medir la flexión dorsal del pie en condiciones de carga.

Se realizó una exploración en sedestación para examinar alteraciones musculoligamentosas, como la debilidad de partes blandas, es decir, hiperlaxitud ligamentosa o hipermovilidad de las articulaciones del primer radio, que producen una hipermovilidad de la articulación metatarsocuneana o cuneoescafoidea en la columna medial, provocando que el primer metatarsiano reciba menos carga.

También se realizaron el test Silverfskiold para valorar el acortamiento de gastrocnemios y el test de Lachman en las articulaciones metatarsofalángicas menores para medir la inestabilidad de dichas articulaciones.

Se efectuó una exploración dermatológica y de deformidades para poder detectar patologías asociadas como hallux valgus, hallux rigidus o callosidades plantares.

c) De manera complementaria se realizó un estudio radiológico del pie con radiografía simple anteroposterior en carga para confirmar e identificar el primer radio corto respecto al segundo o el resto de los metatarsianos centrales, teniendo en cuenta la parábola metatarsal. Es decir, la insuficiencia de primer radio que se sospechaba por la exploración en el estudio biomecánico y se identificó la longitud del primer radio con respecto al segundo de forma visual por el examinador principal del estudio sin utilizar ninguna medición concreta.

En el caso de todos los pacientes se midieron los ángulos del hallux valgus e intermetatarsal, determinando si eran mayores que 9 y 15 grados que son sus valores normales; definiendo el ángulo HV como el ángulo creado por la longitud del eje de la falange proximal del hallux y la del primer metatarsiano y el ángulo IM, que fue definido como el ángulo creado por la longitud del eje del primer y el segundo metatarsianos.

d) La escala AOFAS para valorar el dolor, la función (actividad, distancia máxima caminada, superficie de la marcha, anormalidad del paso, movilidad sagital, movilidad del retropié y estabilidad del tobillo) y la alineación, se utilizó para medir los resultados del estudio y los tratamientos empleados.

\section{Intervenciones realizadas y protocolo de estudio}

Después de evaluar al paciente y determinar todos los factores etiológicos, se inició el tratamiento mediante soportes plantares personalizados con descarga metatarsal, revalorando a los pacientes al mes, a los 3 meses, a los 6 meses y al año, y considerando la efectividad del tratamiento en función del valor de la escala AOFAS repitiéndola también al mes, 3, 6 y 12 meses.

$\mathrm{Si}$ a los 3 meses de tratamiento con soportes plantares el paciente seguía con la sintomatología, es decir, si la puntuación de la escala AOFAS era la misma o inferior, se recomendó por parte del clínico al paciente la infiltración con corticoides, y según la decisión subjetiva de paciente, se procedió a realizarla, con revisión de los pacientes a los 3 meses. Si dado este tiempo persistía dicha sintomatología, se procedió a la cirugía mediante osteotomía simple de Weil.

\section{Tratamiento con soportes plantares}

Según los criterios de diagnóstico anteriores, los pacientes fueron tratados con soportes plantares personalizados con descarga en forma de barra metatarsal y extensión de Morton en el primer metatarsiano, con la finalidad de proporcionar un reparto equitativo de las cargas en la zona del metatarso y descargar la zona de conflicto en los metatarsianos centrales afectados, ya que existen estudios que han mostrado que estas piezas subcapitales, como la extensión de Morton, funcionan debidamente ${ }^{40}$. Se consideran otros dos parámetros que pueden contribuir también a la aparición de la metatarsalgia: la adaptación a medida del arco plantar del paciente y la neutralización de la articulación subastragalina.

Se utilizaron materiales ortopodológicos para la elaboración de los soportes plantares: la base de resinas de 1,2 mm, revestida con un forro de foam microperforado de entre 1,5- 
$3 \mathrm{~mm}$. Se emplearon también dos elementos esenciales para la compensación de la carga y el alivio de la sintomatología: la barra metatarsal y la extensión de Morton. La barra metatarsal retrocapital fue completa de 5-6 $\mathrm{mm}$ de roval foam para descargar las cabezas de los metatarsianos y para extender los dedos desde el borde interno al extremo del antepie. El borde anterior retrocapital a los 5 metatarsianos finalizaba en el primer metatarsiano por detrás de los sesamoideos y el borde posterior por delante de la base de los 5 metatarsianos. La extensión de Morton era de EVA de entre 3-4 mm y densidad de 40 shoreA del primer radio para establecer una magnitud normal de la fuerza reactiva del suelo sobre la primera cabeza metatarsiana, asumiendo el primer metatarsiano su carga normal, y evitando la transferencia de cargas excesivas al segundo metatarsiano.

Se realizó una toma de molde previa con espuma fenólica en semicarga, dibujando los elementos deseados y se especificaron los grosores, los materiales y se mandó al taller Aixalá Sabater Artesá SL, Lleida, España, para su confección y adaptación al paciente.

Todas las prescripciones de los soportes plantares, de los materiales de descarga, así como la toma de moldes, fueron realizadas por el mismo observador, que es el investigador principal del estudio (BCD).

\section{Tratamiento con infiltraciones: corticoides}

Los pacientes que no respondieron al tratamiento con soportes plantares porque el valor de AOFAS era bajo y el paciente continuaba refiriendo dolor y la sintomatología persistía a los tres meses, recibieron una infiltración con corticoides con Celestone Cronodose ${ }^{\circledR}$ (betametasona, fosfato sódico/betametasona, acetato, Merck Sharp \& Dohme, de España, S.A.). Las infiltraciones con corticoides $2 \mathrm{ml} \mathrm{se}$ inyectaron mezcladas con anestésico (2 $\mathrm{ml}$ mepivacaína $2 \%$ ). Se realizó una sola infiltración periarticular, por los efectos secundarios que podía causar siguiendo las recomendaciones del laboratorio que lo produce ${ }^{41}$. Los pacientes también firmaron un consentimiento informado específico. Todas las infiltraciones fueron realizadas por el mismo observador que es el investigador principal del estudio (BCD).

\section{Tratamiento quirúrgico}

Si a los 6 meses los tratamientos conservadores (plantillas más infiltración) resultaban ineficientes, dado que el valor de AOFAS era bajo y el paciente seguía refiriendo dolor, y la sintomatología persistía, los pacientes fueron intervenidos quirúrgicamente mediante la osteotomía de Weil para metatarsianos centrales por cirugía abierta y con osteosíntesis. Siguiendo el protocolo habitual de cirugía ambulatoria, los pacientes firmaron un consentimiento informado oficial y recibieron la profilaxis antibiótica debida, cefazolina $2 \mathrm{~g}$ i.v. Todos los pacientes fueron visitados por un anestesista para determinar la técnica de anestesia, sedación y bloqueo poplíteo o de tobillo, y se pidieron las pruebas complementarias: analítica básica con vitamina D, placa de tórax y ECG. Se usó torniquete de isquemia por encima del tobillo a $250 \mathrm{~mm} \mathrm{Hg}$, con hemostasia por barrido previa. Se planificó la intervención quirúrgica según el estudio de la radiografía y la valoración física prequirúrgica del paciente midiendo los ángulos HV e IM si había HAV asociado y revisando de nuevo el acortamiento del primer metatarsiano respecto al segundo metatarsiano y el resto de la parábola para valorar si convenía realizar correcciones del resto de metatarsianos. En ocasiones se realizaron osteotomías de Weil de varios metatarsianos, en casos de HAV asociado también se realizó técnica quirúrgica sobre él, así como realineaciones de los dedos mediantes artrodesis interfalángicas y alargamientos de tendones o tenotomías.

La osteotomía de Weil se realizó con la finalidad de acortar según lo previsto y descomprimir los metatarsianos pertinentes sobre el extremo distal de uno de los metatarsianos centrales ${ }^{29,42}$. Se realizó un vendaje postoperatorio al paciente sin permitir la deambulación prolongada durante 7 días y deambulando a partir de entonces con una bota de Walker durante 3 semanas más. Todas las intervenciones quirúrgicas fueron realizadas por el mismo observador, que es el investigador principal del estudio (BCD).

\section{Análisis de datos}

El tiempo de seguimiento de todos los pacientes fue de un año desde que entraron en el estudio y se realizaron las pruebas diagnósticas y el primer tratamiento con soportes plantares revisándolos al primer mes, a los 3, 6 y 12 meses. A los pacientes que fueron infiltrados a los 3 meses, se les siguió hasta finalizar el estudio al año, es decir, 9 meses más, con revisiones a los 15 días de la infiltración y a los 3 y 6 meses de la misma. En el caso de los pacientes que fueron intervenidos quirúrgicamente a los 6 meses, el seguimiento fue a 6 meses hasta finalizar el estudio. Aunque se revisaron semanalmente durante 2 meses y mensualmente hasta los 6 meses del postoperatorio.

Las variables dependientes se obtuvieron de los datos físicos, demográficos, radiológicos y patológicos de los pacientes, incluyendo las variables explicativas del tratamiento con soportes plantares, infiltraciones y cirugía.

Se analizaron las variables sexo, edad, índice de masa corporal (dividido en dos categorías: normal o sobrepeso), actividad física (trabajo de más de 5 horas, actividad física deportiva, ambas o ninguna) y patologías asociadas.

La exploración del paciente aportó los datos físicos y las variables del pie que influyen directamente en la metatarsalgia: se analizaron las variables morfología del pie (según el FPI en A "FPI de - 12 a -6", B "FPI de $-5 a+5$ " y C "FPI de +6 $a+12$ "), intervenciones quirúrgicas previas de pie y tobillo (si el paciente había sido intervenido con anterioridad o no), ángulo IM ( $A$ "ángulo $I M<9$ " y $B$ "ángulo $I M \geq 9$ "), y ángulo del hallux valgus ( $A$ "ángulo $H V<15$ " y $B$ "ángulo $H V \geq 15^{\prime \prime}$, retracción de gastrocnemios según el test de 
Lunge para medir la flexión dorsal del pie en condiciones de carga y Silverfkiold Test, respecto a la musculatura posterior y la limitación articular (Sí “tiene retracción” y NO "no tiene retracción"), inestabilidad de la articulación metatarsofalángica afectada (según el test de Lachman para medir la inestabilidad de la articulación metatarsofalángica de la zona del dolor relacionada al plato plantar flexor (normal o inestabilidad), hallux valgus (presencia o no de HAV), uso de zapatos de tacón ("zapatos de tacón" o "no zapatos de tacón"), tipo de insuficiencia de primer radio (según los 4 tipos de insuficiencia de primer radio descritos en la literatura: congénitas, iatrogénicas, partes blandas, pie plano).

La variable independiente escala AOFAS para valorar el dolor, la función (actividad, distancia máxima caminada, superficie de la marcha, anormalidad del paso, movilidad sagital, movilidad del retropié y estabilidad del tobillo) y la alineación, se utilizó para medir los resultados del estudio y los tratamientos empleados.

El test de rango con signo de Wilcoxon (Wilcoxon signed Rank test) se utilizó para la comparación de los resultados de los pacientes que mejoraron, debido a los tratamientos, con los que no mejoraron según los valores de AOFAS.

Se compararon las diferencias de la escala AOFAS entre la primera visita y las visitas de los 3, 6 y 12 meses (Tabla I). Se consideraron resultados significativos a partir de un valor $\mathrm{p}$ menor o igual a 0.05 .

Se aplicó el "t-test" para contrastar la incidencia de las variables en el dolor, función y alineación (AOFAS) de los pacientes antes de ser tratados y después de aplicar cada uno de los tratamientos. Para evaluar el efecto de estas variables y de su influencia en el tratamiento se utilizó un análisis mediante el "test de proporciones" para identificar los factores que fueron potencialmente asociados.

Se aplicaron los test para comparar cada variable con el tratamiento. A los 3 meses comparando los pacientes que han mejorado el dolor con plantillas con los que no y que en consecuencia se trataron con infiltración. A los 6 meses se volvieron a pasar los test, esta vez para comparar los pacientes que mejoraron el dolor o bien con plantillas o bien con infiltración con los que no curaron y fueron operados. Así, las variables que sean significativas tanto a los 3 como a los 6 meses son las que tienen mayor influencia sobre el tratamiento.

Todos los análisis estadísticos se han realizado utilizando el paquete R [R, Developmental, Core, Team. R: A Language and Environment for Statistical Computing 2017; disponible en: http://www.R-project.org ${ }^{43}$.

\section{RESULTADOS}

Un total de 56 pacientes con diagnóstico de metatarsalgia por insuficiencia de primer radio que cumplían los criterios de inclusión fueron incluidos en el estudio y analizados. 41 pacientes $(73.2 \%)$ son mujeres y 15 pacientes $(26.7 \%)$ son hombres. La edad media del grupo fue de 51.25 años; en el caso de las mujeres resultó una edad media de 55 (entre 35-81) y en el caso del grupo de los hombres fue de 47.5 (26-75). Las Tablas I y II exponen los datos demográficos y las variables recogidas en la muestra del estudio.

De los 56 pacientes iniciales en el estudio, a los 3 meses, 46 pacientes $(82.14 \%$ ) habían mejorado y obtuvieron una puntuación superior en la escala AOFAS con soportes plantares personalizados con barra metatarsal. Los 10 pacientes $(18.85 \%)$ que no mejoraron con plantillas, fueron infiltrados de forma periarticular con corticoides.

A los 6 meses, de los 56 pacientes 46 habían mejorado con soportes plantares y 4 (7.14 \%) con la infiltración, los 6 (10.71\%) pacientes donde no funcionó la infiltración ni previamente los soportes plantares, fueron intervenidos quirúrgicamente mediante la osteotomía de Weil.

Se muestran en la Tabla III los resultados de la comparación de los pacientes que mejoraron con los que no mejoraron según los valores de AOFAS en la primera visita, con los valores de AOFAS en las visitas de los 3, 6 y 12 meses, restando la diferencia y otorgando un valor $p$ que en todos los casos fue significativo. Mostrando que los 3 tratamientos mejoraron la puntuación de AOFAS e indicando que la diferencia en cada visita es más grande y, por tanto, los tratamientos mejoran la sintomatología más a largo plazo.

El análisis ${ }^{29}$ estadístico mostró la presencia de asociaciones estadísticamente significativas entre las variables analizadas y la presencia de metatarsalgia por insuficiencia de primer radio antes de comenzar el estudio. La presencia de IMC mayor de 25, la de patologías sistémicas asociadas y la de inestabilidad metatarsofalángicas fueron las únicas variables asociadas a la presencia de metatarsalgia en el periodo pretratamiento.

La Tabla IV muestra el análisis comparativo de las variables que mostraron asociación significativa con la presencia de no mejoría a los 3 meses (después de tratamiento con plantillas) y a los 6 meses (después de tratamiento con plantillas e infiltración).

La presencia de cirugía previa en pie y tobillo fue la única variable que se vio asociada a la presencia de no mejoría y, por tanto, prevalencia de la metatarsalgia a los 3 meses y a los 6 meses.

La Tabla $\vee$ muestra el modelo de regresión logística que identificó una única variable predictiva de no mejoría tras tratamiento con plantillas a los 3 meses y tras tratamiento con plantillas e infiltraciones a los 6 meses. 
Tabla I. Variables recogidas de la muestra del estudio.

\begin{tabular}{|c|c|c|c|c|c|}
\hline & & & & & \\
\hline & & Pacientes & $\%$ Pacientes & Pacientes & $\%$ Pacientes \\
\hline & A & 3 & 5.36 & 4 & 7.14 \\
\hline Actividad & $A B$ & 4 & 7.14 & 17 & 30.36 \\
\hline & B & 6 & 10.71 & 17 & 30.36 \\
\hline & $\mathrm{C}$ & 2 & 3.57 & 4 & 5.36 \\
\hline & NO & 8 & 14.29 & 18 & 32.14 \\
\hline Alteracionec & Sí-A & 2 & 3.57 & 7 & 12.50 \\
\hline Hiteraciones & Sí-B & 1 & 1.79 & 1 & 1.79 \\
\hline & Sí-C & 4 & 7.14 & 15 & 26.79 \\
\hline IO previas & No & 13 & 23.21 & 31 & 55.36 \\
\hline IQ previas & Sí & 2 & 3.57 & 10 & 17.86 \\
\hline Inect Artic & Inestable & 2 & 3.57 & 8 & 14.29 \\
\hline . & Estable & 13 & 23.21 & 33 & 58.93 \\
\hline Retracción & No & 9 & 16.07 & 27 & 48.21 \\
\hline Netractiom & Sí & 6 & 10.71 & 14 & 25.00 \\
\hline 7anato & Sin tacón & 15 & 26.79 & 26 & 46.43 \\
\hline Zapdiv & Tacón & 0 & 0.00 & 15 & 26.79 \\
\hline & Congénita & 15 & 26.79 & 27 & 48.21 \\
\hline In $1^{\text {er }}$ radio & latrogenia & 0 & 0.00 & 8 & 14.29 \\
\hline III. I . Taurio & Parte blanda & 0 & 0.00 & 3 & 5.36 \\
\hline & Pie plano & 0 & 0.00 & 3 & 5.36 \\
\hline HAV & No & 10 & 17.86 & 22 & 39.29 \\
\hline 然 & Sí & 5 & 8.93 & 19 & 33.93 \\
\hline $\mathrm{HR}$ & No & 14 & 25.00 & 41 & 73.21 \\
\hline II & Sí & 1 & 1.79 & 0 & 0.00 \\
\hline
\end{tabular}

En "Actividad": A: es que trabaja más de $5 \mathrm{~h}$ de pie; AB: es que realiza ambas actividades; B: es que realiza actividad deportiva; C: ninguna. En "Alteraciones", patologías asociadas: a: diabéticos; b: tienen alguna patología de columna vertebral asociada; c) tienen otras patologías asociadas, como hipertensión arterial, colesterol u otras. IQ previas: intervenciones quirúrgicas previas. Inest. Artic.: inestabilidad articular. In. 1r. radio: insuficiencia del primer radio. HAV: hallux abductus valgus. HR: hallux rigidus.

\section{Tabla II. Datos demográficos.}

\begin{tabular}{lllllll} 
& Hombres & & \multicolumn{3}{c}{ Mujeres } \\
& Media & Desviación & IC 95 $\%$ & Media & Desviación & IC 95 \% \\
\hline Edad & 47.1 & 14.4 & $39.1-55.1$ & 53.9 & 10.7 & $50.5-57.3$ \\
\hline IMC & 27.0 & 5.1 & $24.2-29.8$ & 25.0 & 4.0 & $23.8-26.3$ \\
\hline FPI & -1.5 & 4.4 & $-3.9-1.0$ & -0.2 & 4.1 & $-1.5-1.0$ \\
\hline Áng. IM & 9.2 & 1.2 & $8.6-9.9$ & 9.4 & 1.2 & $9.1-9.8$ \\
\hline Áng. HV & 13.9 & 2.4 & $12.6-15.2$ & 14.3 & 2.4 & $13.6-15.1$ \\
\hline
\end{tabular}

IMC: índice de masa corporal. FPI: Foot Posture Index. Áng. IM: ángulo intermetatarsal. Áng HV: ángulo hallux valgus.

\section{Tabla III. Resultados de la comparación de la diferencia del valor de AOFAS de los 56 pacientes entre la} primera visita y las visitas a los 3,6 y 12 meses.

\begin{tabular}{|c|c|c|c|c|c|c|c|c|}
\hline Visitas & Pre & & Post & & Diferen & & & \\
\hline Pre-Post & Media & Desviación & Media & Desviación & Media & Desviación & IC $95 \%$ & P-valor \\
\hline $1-3$ & 53.5 & 16.8 & 78.3 & 14.5 & 24.8 & 12.0 & $20.5-28.0$ & $<0.001$ \\
\hline $1-6$ & 53.5 & 16.8 & 84.7 & 15.3 & 31.3 & 14.9 & $27.5-35.5$ & $<0.001$ \\
\hline $1-12$ & 53.5 & 16.8 & 89.0 & 13.8 & 35.5 & 17.6 & $31.5-40.5$ & $<0.001$ \\
\hline
\end{tabular}


Tabla IV. Compara el porcentaje de pacientes que mejoran con soportes plantares a los 3 meses con los que

no mejoran y prevalece la metatarsalgia y a los 6 meses a los pacientes que mejoran con soportes plantares e infiltración con los que no.

\begin{tabular}{|c|c|c|c|c|c|c|}
\hline & \multicolumn{2}{|c|}{$\begin{array}{l}\text { Visita } 3 \text { meses } \\
\text { Prevalencia } \\
\text { metatarsalgia }\end{array}$} & \multirow[b]{2}{*}{$\mathrm{p}$-valor } & \multicolumn{2}{|c|}{$\begin{array}{l}\text { Visita } 6 \text { meses } \\
\text { Prevalencia } \\
\text { metatarsalgia }\end{array}$} & \multirow[b]{2}{*}{ p-valor } \\
\hline & $\mathrm{n}$ & $\%$ & & $\mathrm{n}$ & $\%$ & \\
\hline \multicolumn{7}{|l|}{ Sexo } \\
\hline$M(41)$ & 7 & 17.1 & 1.000 & 5 & 12.2 & 0.917 \\
\hline $\mathrm{H}(15)$ & 2 & 13.3 & & 1 & 6.7 & \\
\hline \multicolumn{7}{|l|}{ IMC } \\
\hline$A(27)$ & 5 & 18.5 & 0.907 & 5 & 18.5 & 0.165 \\
\hline B (29) & 4 & 13.8 & & 1 & 3.4 & \\
\hline \multicolumn{7}{|l|}{ Actividad } \\
\hline$A(7)$ & 2 & 28.6 & 0.408 & 2 & 28.6 & 0.231 \\
\hline$A B(21)$ & 2 & 9.5 & & 1 & 4.8 & \\
\hline$B(23)$ & 5 & 21.7 & & 3 & 13.0 & \\
\hline \multicolumn{7}{|l|}{ Alteraciones } \\
\hline No (26) & 4 & 15.4 & 0.597 & 3 & 11.5 & 1.000 \\
\hline Sí(19) & 5 & 26.3 & & 3 & 15.8 & \\
\hline \multicolumn{7}{|l|}{ Tipo pie } \\
\hline$A(10)$ & 2 & 20.0 & 1.000 & 2 & 20.0 & 0.509 \\
\hline B (43) & 7 & 16.3 & & 4 & 9.3 & \\
\hline \multicolumn{7}{|l|}{ Cirugía previa } \\
\hline No (44) & 4 & 9.1 & 0.023 & 2 & 4.5 & 0.020 \\
\hline Sí (12) & 5 & 41.7 & & 4 & 33.3 & \\
\hline \multicolumn{7}{|l|}{ Ángulo IM } \\
\hline$<9(32)$ & 4 & 12.5 & 0.636 & 2 & 6.2 & 0.418 \\
\hline$>9(24)$ & 5 & 20.8 & & 4 & 16.7 & \\
\hline \multicolumn{7}{|l|}{ Ángulo HV } \\
\hline$<15(32)$ & 4 & 12.5 & 0.636 & 2 & 6.2 & 0.418 \\
\hline$>15(24)$ & 5 & 20.8 & & 4 & 16.7 & \\
\hline \multicolumn{7}{|l|}{ BiHAV } \\
\hline No (32) & 4 & 12.5 & 0.636 & 2 & 6.2 & 0.418 \\
\hline Sí (24) & 5 & 20.8 & & 4 & 16.7 & \\
\hline \multicolumn{7}{|l|}{ Retracción } \\
\hline No (36) & 6 & 16.7 & 1.000 & 4 & 11.1 & 1.000 \\
\hline Sí (20) & 3 & 15.0 & & 2 & 10.0 & \\
\hline \multicolumn{7}{|c|}{ Inestabilidad articular } \\
\hline Inestable (10) & 3 & 30.0 & 0.396 & 2 & 20.0 & 0.629 \\
\hline Estable (46) & 6 & 13.0 & & 4 & 8.7 & \\
\hline \multicolumn{7}{|l|}{ Zapato Talón } \\
\hline No (41) & 6 & 14.6 & 0.942 & 5 & 12.2 & 0.917 \\
\hline Sí (15) & 3 & 20.0 & & 1 & 6.7 & \\
\hline \multicolumn{7}{|l|}{ Insuf-1r } \\
\hline Congénita (42) & 6 & 14.3 & 0.287 & 3 & 7.1 & 0.034 \\
\hline latrogénica (8) & 3 & 37.5 & & 3 & 37.5 & \\
\hline
\end{tabular}

IMC: índice de masa corporal (A: peso normal inferior a 25; B: sobrepeso u obeso superior a 25). Actividad (A: trabaja más de 5 horas de pie; B: realiza actividad deportiva; AB: realiza ambas cosas; C: no realiza ninguna actividad). Tipo de pie (A: el FPI es entre -12 a -6; B: el FPI es entre -5 a +5; C: el FPI es de +6 a +12). Hav: hallux valgus angle. IM: ángulo intermetatarsal. 
Tabla V. Modelo de regresión logística de variables predictivas de no mejoría en pacientes con metatarsalgia por insuficiencia de primer radio.

\begin{tabular}{lllllll}
\multicolumn{3}{c}{ Visita 3 meses } & \multicolumn{5}{c}{ Visita 6 meses } \\
\hline & Odds ratio & IC 95\% & p-valor & Odds ratio & IC 95\% & p-valor \\
\hline Cirugía previa & 0.14 & $0.03-0.6$ & 0.012 & 0.095 & $0.01-0.57$ & 0.013 \\
\hline
\end{tabular}

\section{DISCUSIÓN}

En este trabajo, una vez diagnosticados mediante estudio biomecánico y radiografía simple, se aplicaron los tres tratamientos por orden cronológico y de conservador a quirúrgico: soportes plantares personalizados con barra metatarsal, infiltración con corticoides a los 3 meses si los pacientes no mejoraron y finalmente con cirugía mediante la osteotomía de Weil si a los 6 meses de estudio no funcionaba ninguno de los tratamientos anteriores. Se midió su mejora con la escala AOFAS al valorar el dolor, función y alineación en cada una de las visitas previas y posteriores a cada tratamiento. Igualmente, se han evaluado y considerado diferentes factores etiológicos que podían estar asociados y por tanto influenciar en la aparición de la metatarsalgia por insuficiencia de primer radio y el resultado de los tratamientos, pudiendo ser predictivos.

El concepto de síndrome de la insuficiencia de primer radio fue introducido por A. Viladot, en 1996 en Barcelona, describiéndolo como la disminución de la carga que soportaba la cabeza del primer metatarsiano, provocando una sobrecarga del resto de los metatarsianos. En las últimas décadas se ha avanzado en el conocimiento sobre la mecánica del antepié y sobre los métodos de diagnóstico y tratamiento, en el concepto de metatarsalgia de apoyo y propulsión, de metatarsalgias por transferencia, de su etiología congénita, iatrogénica, debilidad de partes blandas y pie plano. Se han fijado bases más sólidas en cuanto a anatomía de antepié, fórmula metatarsal, sus tratamientos conservadores o quirúrgicos y sus diferentes factores etiológicos y variables asociadas.

Los resultados obtenidos en el presente estudio demuestran que el protocolo de tratamiento aplicado es eficaz desde el punto de vista de mejoría de puntuación en el valor AOFAS de las visitas de los 3, 6 y 12 meses, por la reducción del valor de esta. Se mostró una disminución del dolor, mejoría de la función y alineación. El valor de AOFAS, aunque mira otras cosas, se focaliza más en el dolor en comparativa con otras escalas como SF36 ${ }^{44}$.

Besse $^{3}$ realizó un trabajo determinando las diferentes etiologías de la metatarsalgia, entre ellas la metatarsalgia por insuficiencia de primer radio. El estudio aporta datos de mucha utilidad, ya que comentan los métodos de diagnóstico y todos los tratamientos, aunque no en orden, conservadores hablando de soportes plantares y quirúrgicos. Se enfatiza en la fiabilidad de la osteotomía de Weil en comparación con DMMO y considerando la resección de gastrocnemios o la reparación del plato plantar de manera asociada. Aunque estos autores no inciden en el orden de tratamiento ni realizaron un estudio prospectivo, coincidimos en los tratamientos que se realizan en este estudio excepto las infiltraciones con corticoides y a su vez en la importancia de sopesar los factores biomecánicos asociadas.

En este estudio prospectivo y observacional se profundiza en la etiología de la metatarsalgia por insuficiencia de primer radio únicamente, y se aplican los tres tratamientos que se han considerado más válidos de conservador a quirúrgico y se efectúa un seguimiento con la escala AOFAS en las diferentes visitas. Se considera que el tratamiento conservador más eficaz son los soportes plantares con descarga metatarsal y extensión de Morton ${ }^{40}$, encontrando en la literatura diferentes estudios que lo avalan. Postema y cols. ${ }^{17}$ analizan la influencia de los soportes plantares personalizados en cuanto a las presiones plantares, obteniendo que producen una disminución del pico de presión y de la fuerza de impulso. Holmes y Timmerman ${ }^{14}$ llegan a la misma conclusión, analizando el efecto de las descargas en las presiones plantares baropodométricas. Williams ${ }^{15}$ considerando, al igual que Burns y cols. ${ }^{16}$ en los pies cavos, que los soportes plantares pueden corregir la biomecánica de la metatarsalgia, obteniendo que los pacientes con soportes plantares personalizados con descarga metatarsal mejoran más las presiones plantares que las plantillas estándar.

Diferentes estudios han mostrado la mayor eficacia de las ortesis plantares a medida en la reducción de las presiones plantares y de la sintomatología clínica sobre placebo o plantillas estándar u otros tipos de tratamiento ${ }^{26,27}$. Otros estudios muestran que la extensión de Morton funciona mejor que las piezas retrocapitales en reducción de las presiones plantares durante la marcha ${ }^{40}$. Estos autores dan valor a la importancia del uso de soportes plantares en la metatarsalgia provocada por insuficiencia de primer radio, como se muestra en nuestro estudio. Los resultados demuestran que en el inicio existe una mejora clínica significativa respecto a la reducción de los síntomas de la patología en 46 pacientes que mejoraron con soportes plantares. 
En cuanto a las infiltraciones con corticoides, varios autores $^{18,19}$ las consideran un tratamiento válido en las partes blandas inflamadas después de una sobrecarga, así como metatarsalgias estáticas en la inestabilidad de las articulaciones metatarsofalángicas, sobre todo en casos de intensas reacciones inflamatorias locales. Los resultados en este estudio, utilizando las infiltraciones con corticoides de manera complementaria si no tenía éxito terapéutico el soporte plantar, fueron también significativos. Los resultados muestran que, de los 10 pacientes que se infiltraron a los 3 meses, 4 de ellos mejoraron su sintomatología respecto a la reducción del valor de la escala AOFAS. De este modo, lo consideramos como un tratamiento válido asociado a los soportes plantares cuando, después de utilizar estos, permanece el edema y la inflamación de partes blandas.

El tratamiento quirúrgico es más frecuente en la literatura, a pesar de sus controversias y comparaciones entre técnicas, generalmente indicado para metatarsalgias recalcitrantes refractarias a los tratamientos conservadores. Actualmente, la osteotomía de Weil es la más utilizada por su estabilidad y sencillez de realizar en metatarsalgias por insuficiencia de primer metatatarsiano.

Barouk $^{5}$, en su estudio sobre la recurrencia de la metatarsalgia, apunta que en la recurrencia de la metatarsalgia de radios medios, la cirugía de hallux valgus puede ser un problema por dos motivos: por la posición del primer metatarsiano después de una inapropiada corrección, o por no reconocer un acortamiento de gastrocnemios previo a la cirugía. El autor preconiza que el mejor tratamiento es restaurar la normalidad de la anatomía y que la cirugía en los radios afectados puede ser la solución. Se centra básicamente en que la recurrencia de la metatarsalgia tiene un origen multifactorial y puede ser considerada como una iatrogenia o un fallo de una cirugía previa. Como significativamente observamos en nuestro estudio, donde encontramos como única variable asociada a la recurrencia de la metatarsalgia por insuficiencia de primer radio, los pacientes que fueron intervenidos quirúrgicamente con anterioridad a los 3 meses de tratamiento. A los 6 meses también fue significativa junto a insuficiencia de primer radio por iatrogenia respecto a los pacientes que tenían una insuficiencia congénita o por pie plano, significando que estaban asociadas a la recurrencia de la metatarsalgia y, por tanto, los pacientes tuvieran que ser intervenidos quirúrgicamente.

Así se ha reflejado también en el resultado de este estudio, ya que 6 de los 56 pacientes, a los 6 meses, no mejoraron ni con soportes plantares ni con infiltración. Fueron intervenidos quirúrgicamente, observando en la revisión a los 12 meses una mejora significativa en el valor de AOFAS ente los pacientes al inicio y al final del estudio.

En cuanto a los factores etiológicos, antes de tratar a los pacientes y las variables asociadas a la metatarsalgia por insuficiencia de primer radio que podían influir en el resultado terapéutico de los diferentes tratamientos, se halló que tres variables fueron significativas: la presencia de IMC mayor de
25, la presencia de patologías sistémicas asociadas y la presencia de inestabilidad metatarsofalángica (Tabla I).

En este estudio la influencia del IMC ha sido una variable significativa en los pacientes antes de ser tratados. Se ha obtenido que pacientes con sobrepeso u obesos tienen valor de AOFAS más bajo y en consecuencia un IMC alto incide en la metatarsalgia por insuficiencia de primer radio. Contrastado también en otros estudios consultados como el de Butterworth y cols. ${ }^{45}$ y Dufour y cols. ${ }^{46}$ que resaltan la evidencia de una fuerte asociación entre el IMC elevado y un dolor en el pie. Hsu y cols. ${ }^{47}$ comparan pacientes diabéticos de tipo 2 con pacientes sanos, determinando que los tejidos plantares de debajo de las cabezas de los metatarsianos suelen estar alterados a la vez que reciben carga.

La inestabilidad de la articulación metatarsofalángica afectada también fue un factor asociado significativo respecto a las que sí eran estables antes de aplicar los tratamientos, ya que encontramos que los pacientes con inestabilidad metatarsofalángica tenían una puntuación de AOFAS más baja. Otros estudios prospectivos, como el de Nery y cols. ${ }^{48}$, tratan de manera muy concreta y específica, similar a este estudio, la evaluación de un protocolo para el tratamiento quirúrgico de las lesiones en las articulaciones metatarsofalángicas de radios medios y las lesiones del plato plantar. Sus hallazgos, como en el presente estudio, demuestran que la inestabilidad de las articulaciones metatarsofalángicas menores es una causa común de dolor y deformidad. Los resultados del estudio de Nery y cols. ${ }^{48}$ presentan que los pacientes con menos alteración o sin alteración de la placa plantar, mirando la inestabilidad de la articulación respectiva, tenían menos dolor con una puntuación de la escala AOFAS más alta.

La única variable significativa a los 3 meses del estudio fue que los pacientes hubieran sido intervenidos quirúrgicamente de pie o tobillo previamente. Se comparó a los pacientes que habían mejorado con soportes plantares con los que no habían mejorado y se observó que una previa intervención influencia en la efectividad del tratamiento. En concreto, es un factor asociado a que los soportes plantares no funcionen, repitiéndose como variable significativa también en las visitas de los 6 meses.

Este hallazgo coincide con el de Maceira y Monteagudo ${ }^{49} \mathrm{y}$ con Barouk ${ }^{5}$, los cuales indican que, en el manejo de la metatarsalgia de transferencia después de la cirugía de hallux valgus, es importante una comprensión profunda de los trastornos anatómicos, para planificar el tratamiento adecuado. Una historia detallada y un examen clínico junto con estudios de imagen permitirán determinar qué salió mal y por qué.

Como limitación del estudio, se indica, en primer lugar, que se dispuso de un bajo número de pacientes. El número total de pacientes fue 56 y el número de pacientes con cada uno de los tratamientos, a consecuencia, fue escaso. Se contó con 46 pacientes tratados con plantillas donde podemos obtener y comparar más datos, pero solo 10 pacientes fueron infiltrados y únicamente 6 pacientes intervenidos quirúrgicamente. El hecho de disponer de un número bajo de pacientes influye 
en la significación de las variables a lo largo del estudio. Otra limitación achacable al presente estudio fue el solamente tener un seguimiento del paciente en un año desde el inicio del primer tratamiento y, por tanto, solamente 6 meses en los pacientes que han sido intervenidos quirúrgicamente. Se interpreta que estudiar y observar a los pacientes más tiempo podría cambiar los resultados y darnos más datos. En último lugar, aunque se ha estudiado un número considerable de variables, se cree que indagando en otras variables no estudiadas, se podría conseguir más significación y la respuesta de por qué funcionan o no los tratamientos.

En conclusión, el presente estudio muestra que es necesario realizar los tratamientos por orden de conservador a quirúrgico y por orden cronológico. La mayoría de casos de metatarsalgia por insuficiencia de primer radio se resuelven con tratamiento conservador, mediante soportes plantares personalizados con barra metatarsal. La infiltración es efectiva en un $40 \%$ de los casos en los cuales el tratamiento con soportes plantares ha fracasado, siendo la cirugía la única vía correctiva en caso del fracaso de los dos tratamientos anteriores. Después de aplicar los tratamientos, a los 3 y a los 6 meses, es sumamente importante tener en cuenta si los pacientes han sido previamente intervenidos de pie y tobillo. La prevalencia de la metatarsalgia en pacientes con insuficiencia de primer radio puede depender de esta variable cuando los tratamientos con soportes plantares no sean efectivos $y$, por tanto, haga falta infiltrarlos. De la misma manera, en pacientes que vayan a ser intervenidos con la osteotomía de Weil porque no han funcionado los tratamientos conservadores previos. Para tratar la patología de la metatarsalgia central por insuficiencia de primer radio, se aconseja a los facultativos que se centren en los tres tratamientos: soportes plantares, infiltración o cirugía con osteotomía de Weil por orden cronológico y de conservador a quirúrgico. Asimismo, se aconseja examinar teniendo en cuenta las variables significativas antes de tratar a los pacientes, y después de cada uno de los tratamientos.

\section{BIBLIOGRAFÍA}

1. Dockery GL. Evaluation and treatment of metatarsalgia and keratic disorders. En: Myerson MS, ed. editor. Foot and ankle disorders. Philadelphia: Saunders Company; 2000. p. 359-77.

2. Campillo MA, Ibáñez SZF. Las metatarsalgias. Rev Esp Reumatol. 2003;30(9):467-538.

3. Besse JL. Metatarsalgia. Orthop Traumatol Surg Res. 2017;103(Suppl 2):S29-39. DOI: 10.1016/j.otsr.2016.06.020.

4. Slullitel G, López V, Calvi JP, Seletti M, Bartolucci C, Pinton G. Effect of first ray insufficiency and metatarsal index on metatarsalgia in hallux valgus. Foot Ankle Int. 2016;37(3):300-6. DOI: 10.1177/1071100715615323.

5. Barouk P. Recurrent metatarsalgia. J Foot Ankle Surg. 2014;53(2):131-6. DOI: 10.1053/j.jfas.2013.12.018.

6. O'Kane C, Kilmartin TE. The surgical management of cetralmetatarsalgia. Foot Ankle Int. 2002;23(5):415-9. DOI: 10.1177/107110070202300508.

7. Mann RA, Chou LB. Surgical management for intractable metatarsalgia. Foot Ankle Int. 1995;16(6):322-7. DOI: 10.1177/107110079501600602.
8. ViladotA. En:Masson, ed. Biomecánica, medicinay cirugía del pie: Síndrome de insuficiencia del primer radio. Barcelona: Masson; 1997. p. 217-25.

9. Viladot-Voegeli A, Viladot-Pericó A, Núñez-Samper Pizarroso MLA. En: En: Masson, ed. Biomecánica, medicina y cirugía del pie: Síndrome de insuficiencia del primer radio. 2. ${ }^{\text {a }}$ ed. Barcelona; 2006. p. 235-48.

10. Christensen J, Jennings $M$. Normal and Abnormal Function of the First Ray. Clin Podiatr Med Surg. 2009;26(3):355-71. DOI: 10.1016/j. cpm.2009.03.004.

11. Gregg J, Schneider T. Marks P MR Imaging and Ultrasound of Metatarsalgia-The Lesser Metatarsals. Radiol Clin North Am. 2008;46(6):106178. DOI: 10.1016/j.rcl.2008.09.004.

12. Yakel J. A guide to orthotic treatment for metatarsalgia. Pod Today. 2012;25(4):74-8.

13. Muñoz D. Tratamiento fisioterápico en complicaciones postquirúrgicas en el pie. REDUCA (Enfermería, Fisioter y Podol). 2012;4(4):54-69.

14. Holmes GB Jr, Timmerman L. A quantitative assessment of the effect of metatarsal pads on plantar pressures. Foot ankle Int. 1990;11(3):141-5. DOI: 10.1177/107110079001100304.

15. Williams B. Can orthotics address the Faulty Biomechanics of metatarsalgia? Pod Today. 2005;18(6):28-32.

16. Burns J, Crosbie J, Ouvrier R, Hunt A. Effective orthotic therapy for the painful cavus foot: a randomized controlled trial. J Am Podiatr Med Assoc. 2006;96(3):205-11. DOI: 10.7547/0960205.

17. Postema K, Burm PE, Zande ME LJ. Primary metatarsalgia: the influence of a custom moulded insole and a rockerbar on plantar pressure. Proshet Orthor Int. 1998;22(1):35-44. DOI: 10.3109/03093649809164455.

18. DiligentJ. Metatarsalgias estáticas. EMC- Podol. 2014;16(1):1-13. DOI: 10.1016/S1762-827X(14)66674-8.

19. Acerboni F, Marcano FA, Gonzalez JA. Causas poco frecuentes de dolor en antepié: a propósito de 4 casos. Rev del pie y tobillo. 2017;31(1). DOI 10.24129/j.rpt.3101.fs 1607016

20. Chapelle C. Intra-articular injections. Rev Med Brux. 2015;36(4):281-7.

21. Dockery GL. The treatment of intermetatarsal neuromas with $4 \%$ alcohol sclerosing injections J Foot Ankle Surg. 1999;38(6):403-8. DOI: 10.1016/S1067-2516(99)80040-4.

22. Jahssr MH, ed. Biomechanics. Philadelphia:WBSaunders Co; 1982. p. 36-67.

23. Cracchiolo A. Office practice footwear and orthotic therapy. Foot Ankle. 1982;2(4):242-8. DOI: 10.1177/107110078200200412.

24. Kotwick JE. Biomechanics of the foot and ankle. Clin Sport Med. 1982;1(1):19-34.

25. Masson, ed. Monografías médico-quirúrgicas del aparato locomotor: Pie degenerativo: clínicay tratamiento médico. Barcelona: Masson; 1997. p. 49-60.

26. Doxey GE. Management of metatarsalgia with foot orthotics. J Orthop Sport Phys Ther. 1985;6(6):324-33. DOI: 10.2519/jospt. 1985.6.6.324.

27. Kang JH, Chen MD, Chen SC. Correlations between subjective treatment responses and plantar pressure parameters of metatarsal pad treatment in metatarsalgia patients: a prospective study. BMC Musculoskelet Disord. 2006;7:95. DOI: 10.1186/1471-2474-7-95.

28. Pawelka S, KopfAZE. Comparison of two insole materials using subjective parameters and pedobarography. Clin Biomech. 1997;12(3):S6-7. DOI: 10.1016/S0268-0033(97)88314-3.

29. Pascual Huerta J, Arcas Lorente C, García Carmona FJ. The Weil osteotomy: A comprehensive review. Rev Esp Podol. 2017;28(2):e38-51. DOI: 10.1016/j.repod.2017.10.003.

30. Roukis TS. Central metatarsal head-neck osteotomies: Indications and operative techniques. Clin Pod Med Surg. 2005;22:197-225. DOI: 10.1016/j.cpm.2004.10.003.

31. Barouk LS. Weil's metatarsal osteotomy in the treatment of metatarsalgia. Der orthopade. 1996;25(4):338-44. DOI: 10.1007/ s001320050034.

32. Munuera P. En: El Primer Radio: biomecánica y ortopodología. Santander: Exa editores, SL; 2009

33. Federer AE, Tainter DM, Adams SB, Schweitzer KM. Conservative Management of Metatarsalgia and Lesser Toe Deformities. FootAnkle Clin. 2018;23(1):9-20. DOI: 10.1016/j.fcl.2017.09.003.

34. Hähni M, Hirschmüller A, Baur H. The effect of foot orthoses with forefoot cushioning or metatarsal pad on forefoot peak plantar pressure in running. J Foot Ankle Res. 2016;9:44. 
35. Sanfélix Genovés J, Giner Ruiz V, Fluixá Soria J, Fuerte Fortea A. Manual de infiltraciones en Atención Primaria. Valencia: Generelitat. Conselleria de sanitat; 2007

36. Redfern D. Treatment of Metatarsalgia with Distal Osteotomies. Foot Ankle Clin. 2018;23(1):21-33. DOI: 10.1016/j.fcl.2017.09.004.

37. Zirm RJ. En: McGlamry's comprehensive textbook of foot and ankle surgery: Indications and Technique of the Weil Osteotomy. Southerland T (ed.). Philadelphia: Wolters Kluwer Health; 2013. p. 224-8.

38. Zirm RJ. The Weil Lesser Metatarsal osteotomy. En: Williams and Wilkins (ed). McGlamry's Comprehensive Textbook of Foot \& Ankle Surgery. 4. ${ }^{a}$ ed. Baltimore: Williams and Wilkins; 2010. p. 224.

39. Trnka HJ, Gebhard C, Mühlbauer M, Ivanic G, RitschI P. The Weil osteotomy for treatment of dislocated lesser metatarsophalangeal joints: Good outcome in 21 patients with 42 osteotomies. Acta Orthop Scand. 2002;73(2):190-4. DOI: 10.1080/000164702753671795.

40. Lorca Navarro O. Influencia de la extensión de Morton en las presiones del antepié. 2016. Disponible en: http://diposit.ub.edu/dspace/handle/2445/102421.

41. Sanitarios. AE de M y P. IMA: Centro de Información online de Medicamentos de la AEMPS. [acceso May 2019] Disponible en: http://www. aemps.gob.es/cima.

42. Downey MS, McGlamry MC. Transverse plane digital deformities. En: Southerland T. editor. McGlamry's comprehensive textbook of foot and ankle surgery. $4^{\circ}$ ed. Philadelphia: Wolters Kluwer Health; 2013. p. 202-23.
43. R: A Language and Environment for Statistical Computing [Internet]. Viena, Austria; 2017. [acceso Jun 2019] Disponible en: http:// www.r-project.org.

44. SooHoo NF, Shuler M, Fleming LL. Evaluation of the validity of the AOFAS clinical rating systems by correlation to the SF-36. Foot Ankle Int. 2003;24(1):50-5. DOI: 10.1177/107110070302400108.

45. Butterworth PA, Landorf KB, Smith SE, Menz HB. The association between body mass index and musculoskeletal foot disorders: A systematic review. Obes Rev. 2012;13(7):630-42. DOI: 10.1111/j.1467789X.2012.00996.x.

46. Dufour AB, Losina E, Menz HB, LaValley MP, Hannan MT. Obesity, foot pain and foot disorders in older men and women. Obes Res Clin Pract. 2017;11(4):445-53. DOI: 10.1016/j.orcp.2016.11.001

47. Hsu CC, Tsai WC, Shau YW, Lee KL, Hu CF. Altered energy dissipation ratio of the plantar soft tissues under the metatarsal heads in patients with type 2 diabetes mellitus: A pilot study. Clin Biomech. 2007;22(1):67-73. DOI: 10.1016/j.clinbiomech.2006.06.009.

48. Nery C, Coughlin M J, Baumfeld D, Raduan FC, Mann TS, Catena F. Prospective evaluation of protocol for surgical treatment of lesser MTP joint plantar plate tears. Foot Ankle Int. 2014;35(9):876-85. DOI: 10.1177/1071100714539659.

49. Maceira E, Monteagudo M. Transfer metatarsalgia post hallux valgus surgery. Foot Ankle Clin. 2014;19(2):285-307. DOI: 10.1016/j. fcl.2014.03.001. 